Review

\title{
Lipid- and Polymer-Based Nanostructures for Cancer Theranostics
}

\author{
Brian T. Luk, Ronnie H. Fang, and Liangfang Zhang ${ }^{\bowtie}$ \\ Department of NanoEngineering and Moores Cancer Center, University of California, San Diego, La Jolla, CA 92093, USA. \\ $\triangle$ Corresponding author: Email: zhang@ucsd.edu, Tel: +1-858-246-0999 \\ (C) Ivyspring International Publisher. This is an open-access article distributed under the terms of the Creative Commons License (http://creativecommons.org/ \\ licenses/by-nc-nd/3.0/). Reproduction is permitted for personal, noncommercial use, provided that the article is in whole, unmodified, and properly cited.
}

Received: 2012.03.19; Accepted: 2012.06.15; Published: 2012.12.10

\begin{abstract}
The relatively new field of nanotheranostics combines the advantages of in vivo diagnosis with the ability to administer treatment through a single nano-sized carrier, offering new opportunities for cancer diagnosis and therapy. Nanotheranostics has facilitated the development of nanomedicine through direct visualization of drug blood circulation and biodistribution. From a clinical perspective, nanotheranostics allows therapies to be administered and monitored in real time, thus decreasing the potential of under- or over-dosing and allowing for more personalized treatment regimens. Herein, we review recent development of nanotheranostics using lipid- and polymer-based formulations, with a particular focus on their applications in cancer research. Recent advances in nanotechnology aimed to combine therapeutic molecules with imaging agents for magnetic resonance imaging, radionuclide imaging, or fluorescence imaging are discussed.
\end{abstract}

Key words: nanotheranostics, liposomes, polymeric nanoparticles, magnetic resonance imaging, radionuclide imaging, fluorescence imaging

\section{Introduction}

Cancer is currently the third most common cause of death in the world after heart and infectious diseases. While the search for efficacious anti-cancer therapeutics continues, it becomes increasingly clear that the accurate and informative visualization of anti-cancer agents following their administration can play an essential role to formulate effective treatment plans [1]. In this regard, nanotheranostics, which combines diagnostic and therapeutic modalities into a single nano-sized carrier, has emerged [2-4]. Such combination allows physicians to monitor the type and quantity of drugs while they are given to each patient, together with other advantages such as assessing drug distribution and release, evaluating drug response and efficacy, and assisting on-demand drug release, all in a non-invasive and real-time fashion. Such information in turn can better assist physicians to tailor treatment plans based on the patient's indi- vidual needs [5], hence potentially lowering the chances of both over and under treatments that have often resulted in adverse side effects in conventional treatments [6]. Indeed, information obtained by combining diagnostic and therapeutic modalities has aided scientists and engineers to design novel anti-cancer drugs as well as optimize existing drug formulations [7].

Among various nano-sized delivery vehicles, liposome and polymeric nanoparticle (NP) are two popular platforms for nanotheranostics (Figure 1). Extensive knowledge has been obtained on the exploitation of these platforms in cancer therapy, particularly their abilities to enhance the efficacy over free drugs through improved drug encapsulation, prolonged circulation half-life, and sustained or triggered drug release [8-10]. In addition, preferential accumulation of liposomes and polymeric NPs at the 
disease sites, as the result of passive targeting through enhanced permeability and retention (EPR) effect and active targeting through selected surface ligands, has been extensively explored [11, 12]. As a result, a number of approved therapeutics based on liposomes and polymeric NPs have entered clinical use, and more are under various stages of clinical development [13-16].

Such progress achieved in developing therapeutic liposomes and polymeric NPs allows researchers to further incorporate imaging modalities commonly used in clinic into these nanocarriers, such as magnetic resonance imaging (MRI) [17], radionuclide imaging [18], and fluorescence imaging [19] (Table 1). Specifically, MRI detects the radiofrequency signal given off as protons return to thermodynamic equilibrium after being pulsed. It offers high spatial resolution without tissue-penetrating limitations. Technical development in MRI is largely focused on further improving the sensitivity, lowering the cost, and reducing the imaging time. Meanwhile, radionuclide imaging by detecting the gamma rays or subatomic particles emitted by injected radioactive nuclei has become an indispensible tool in clinic to aid the evaluation of the development state of many diseases. In particular, single photon emission computed tomography (SPECT) and positron emission tomography (PET) are two major radionuclide-based imaging modalities. A detailed comparison of these two tech- niques requires case-by-case considerations from both biology and physics, which is beyond the scope of this review [20]. Nevertheless, similar to MRI, radionuclide imaging has high sensitivity with no tissue-penetrating limitations. Different from both MRI and radionuclide imaging, fluorescence imaging utilizes photons of characteristic wavelength to excite fluorescent molecules or quantum dots (QDs); the excited, unstable electronic state ultimately causes the emission of light at a higher wavelength that can be detected in the ultraviolet to near infrared range. In addition to the role as an imaging agent, some photosensitizers generate toxic radicals upon excitation, hence capable of dynamic photothermal therapy (DPT)[21]. Optical imaging offers a high sensitivity and the capability of multicolor imaging; however, poor tissue penetration and low spatial resolution have largely limited the use of optical imaging in shallow tissues or small animals.

Current development of nanotheranostics leverages the aforementioned imaging modalities and incorporates them into various NP platforms along with different cancer therapeutics, hence providing novel multimodal platforms with significant clinical application potential. Herein, we review current nanotheranostics that combine anti-cancer therapeutics with MRI, radionuclide imaging, or optical imaging agents, with a primary focus on platforms using liposomes and polymers.
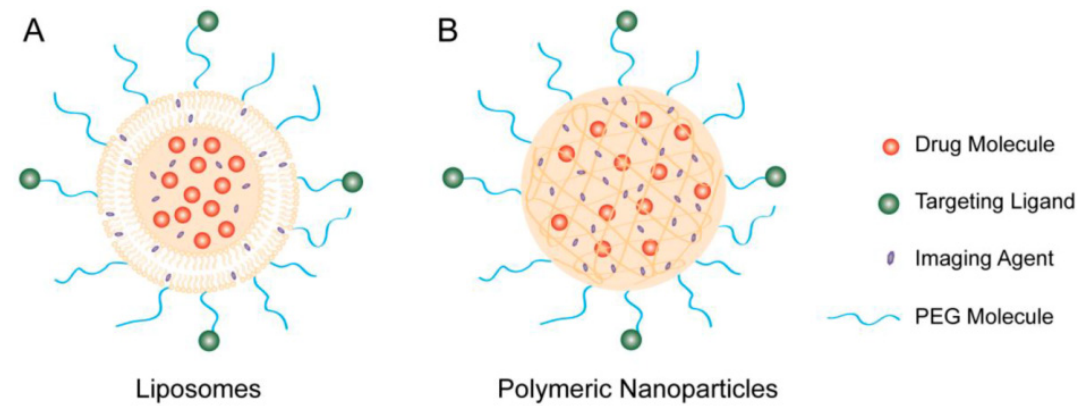

Polymeric Nanoparticles

Figure I. Therapeutic molecules and imaging agents can be co-encapsulated into (A) liposomes or (B) polymeric nanoparticles to form nanotheranostic platforms. Depending on the amphiphilicity of the imaging agents, they can be encapsulated either inside of the liposome or in the lipid bilayers. In addition, the particle surface can be conjugated with targeting ligands for targeted delivery.

Table I. A summary of major imaging modalities used in nanotherapeutics (adapted from reference [9])

\begin{tabular}{|c|c|c|}
\hline Techniques & Pros & Cons \\
\hline Fluorescence Imaging & $\begin{array}{l}\text { * High-throughput screening for target confirmation and compound optimization } \\
\text { * High sensitivity } \\
\text { * Multi-color imaging }\end{array}$ & $\begin{array}{l}\text { * Limited clinical translation } \\
\text { * Low depth penetration }\end{array}$ \\
\hline MRI & $\begin{array}{l}\text { * Clinical translation } \\
{ }^{*} \text { High resolution and soft-tissue contrast }\end{array}$ & $\begin{array}{l}{ }^{*} \text { High cost } \\
{ }^{*} \text { Long imaging time }\end{array}$ \\
\hline PET Imaging & $\begin{array}{l}{ }^{*} \text { Clinical translation } \\
{ }^{*} \text { High sensitivity with unlimited penetration }\end{array}$ & ${ }^{*}$ High cost \\
\hline SPECT Imaging & $\begin{array}{l}{ }^{*} \text { Clinical translation } \\
{ }^{*} \text { Unlimited penetration }\end{array}$ & ${ }^{*}$ Limited spatial resolution \\
\hline
\end{tabular}




\section{Liposome-based nanotheranostics}

Liposomes are established drug carriers for cancer therapy [22-24]. They are closed spherical vesicles composed of a lipid bilayer made of either synthetic or natural phospholipids with diameters around $100 \mathrm{~nm}$. Those with single bilayers are generally synthesized by the hydration of thin lipid films followed by either physical extrusion or sonication [25]. Synthesis methods result in an aqueous phase occupying the center of the liposome, in which hydrophobic and hydrophilic drugs can be encapsulated via a variety of methods [26]. Liposomes have been widely used as delivery vehicles because of their unique abilities to encapsulate both therapeutic and diagnostic agents, protect the encapsulated agents from external environments, prolong systemic circulation lifetime of the encapsulated agents, and be functionalized with various targeting ligands for cellor tissue-specific delivery [16]. Given that some liposome-based therapies such as Doxil ${ }^{\circledR}$ have already been approved for the treatment of certain cancers, loading these carriers with both drug and imaging moieties is a promising strategy to develop next-generation nanotheranostic platforms.

\section{I. Liposome-based therapeutics and MRI}

Liposomes can be loaded with a variety of contrast agents for MRI applications. In particular, superparamagnetic iron oxide NPs (SPIOs) [27-29], gadolinium-, and manganese-based compounds [27, 30,31 ] have been extensively studied for their ability to enhance the contrast in T2-weighted MRI for better in vivo visualization. These agents, together with the therapeutic agents loaded into liposomes, have led to a variety of nanotheranostic particles. For example, Erten et al. have encapsulated iron oxide into a cholesterol/DOPE/DSPC liposome to form a multifunctional nanotheranostic platform [32]. In this formulation, Doxorubicin (DOX) was loaded into a dextran hydrogel placed between the iron oxide core and the lipid shell. The theranostic NPs were studied in athymic nude mice bearing Lewis lung carcinoma xenograft and MIA PaCa-2, a pancreatic cancer xenograft. Observed through a dorsal skinfold window chamber, tumor blood vessels showed enhanced contrast in T2-weighted MRI, owing to the co-localization of the NPs with the tumor vessels. In addition to the role played by iron oxide NPs as contrast agents, the iron oxide NPs also served as a heating source upon the exposure of alternative magnetic field (AMF). Therefore, iron oxide NPs provide a platform for localized hyperthermia-induced drug release, an additional benefit to MRI alone [33]. Gadolinium-based organic agents also showed similar thermosensitive properties. Tagami et al. have formulated a hyperthermia activated-cytotoxic (HaT) liposome composed of DPPC and Brij surfactant, which encapsulated both DOX and the contrast agent gadolinium-diethylenetriaminepentaacetic acid (Gd-DTPA) [34]. The release rate of DOX was monitored by measuring the MR T1 relaxation time, as both parameters were dependent on temperature change. In BALB/c mice implanted with EMT-6 cells, upon heating, an accelerated T1 was correlated with the increased DOX release from the NPs. A higher DOX uptake and a better tumor regression were observed with the heated tumor. Using a similar approach and adding poly(2-ethoxy(ethoxyethyl)vinyl ether), a thermosensitive polymer, into the liposome formulation, liposomes combining temperature-triggered drug release and MRI functions were developed [35]. The liposomes retained DOX in their interior below physiological temperature but released DOX immediately at temperatures above $40{ }^{\circ} \mathrm{C}$. On colon 26 tumor-bearing mice, tumor growth was strongly suppressed when the tumor was heated mildly at $44{ }^{\circ} \mathrm{C}$ for $10 \mathrm{~min}$ at the 8-hour point post-liposome injection. MRI functions were also used to study the dependence of liposome tumor accumulation on liposome size and tumor size. In summary, these studies showed that encapsulating MRI contrast agents such as iron oxide or gadolinium-based compounds into liposomes, together with therapeutic agents, provides a useful approach to developing advanced nanotheranostics with noninvasive MRI.

\subsection{Liposome-based therapeutics and radio- nuclide imaging}

Liposomes can directly encapsulate radionuclide agents to form nanotheranostics with radionuclide imaging. Soundararajan et al. have formulated radiolabeled liposomes by directly loading ${ }^{186} \mathrm{Re}$ and DOX into liposome interior for cancer chemoradionuclide therapy (Figure 2)[36]. In male nude rats bearing xenografts of head and neck squamous cell carcinoma, the liposome formulation showed a prolonged circulation time and decreased liver accumulation. This study also suggests that combining radionuclides with chemotherapeutic drugs not only allows for real-time imaging but also increases efficacy conferred by chemoradionuclide therapy. To ensure a high radionuclide-to-drug ratio inside the liposome following the encapsulation of their mixtures, efforts have been made to fine tune the lipophilicity of the radionuclides through chemical modification of their molecular structures. As a result, a number of radionuclide complexes have been developed with high 
labeling efficiency [37-39]. So far, a large number of radionuclides have been explored to formulate liposome-based theranostic particles, including iodine-131, lutetium-177, yttrium-90, technetium-99m, indium-111, rhenium-166, and rhenium-168 [37, 40-45].

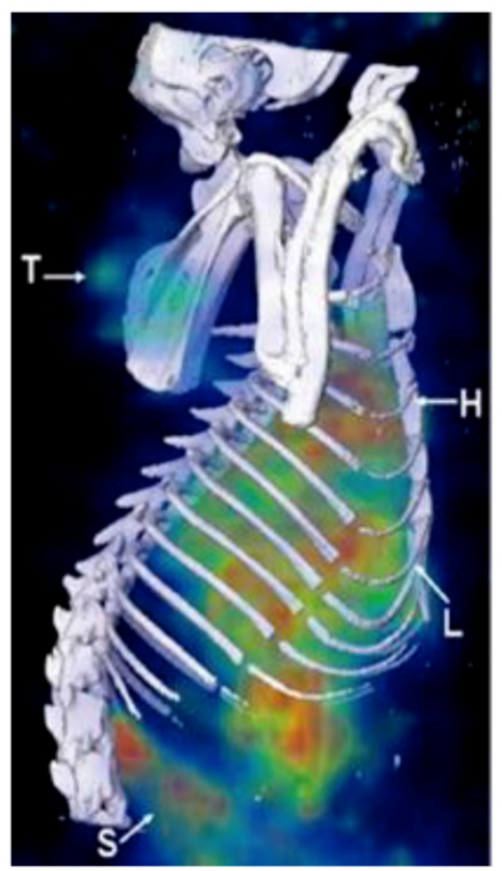

Figure 2. MicroSPECT/CT images acquired at 20 hours post administration of ${ }^{186} \mathrm{Re}-\mathrm{Doxil}$ using a multi-pinhole collimator. Three dimensional (3D) volume rendered SPECT image of 186Re-Doxil overlaid with CT isosurface displayed in bone window shows the accumulation in Tumor ( $T$ ), liver $(L)$, spleen $(S)$ and circulation through heart $(\mathrm{H})$. (Adapted from reference [36]).

In addition, to further enhance image contrast, efforts have been made to explore the potential of PET imaging for nanotheranostics by formulating liposomes containing PET radionuclides. For example, Petersen et al. have developed a polyethylene glycol (PEG)-lipid based liposome formulation loaded with a copper-radionuclide $\left({ }^{64} \mathrm{Cu}\right)$ suitable for PET imaging [46]. Pharmacokinetics of this formulation were studied in female nude mice implanted with human colon adenocarcinoma (HT29) tumor cells in flanks. The accumulation of ${ }^{64} \mathrm{Cu}$-liposomes in tumor was found to be several orders of magnitude higher than that of free ${ }^{64} \mathrm{Cu}$-DOTA. In addition, ${ }^{64} \mathrm{Cu}$-liposomes remained in the blood over a 24-hour period of time, indicating the long circulating nature of the PEGylated liposomes. Meanwhile, the ${ }^{64} \mathrm{Cu}$-liposomes created a high tumor-to-muscle signal ratio in the PET scans comparable to other liposome-based formulations used for SPECT imaging with regular gamma emitters. To further improve the imaging capability, the nanotheranostic liposomes can be further modified by conjugating targeting ligands to their surfaces. For example, monoclonal antibody $2 \mathrm{C} 5$ (mAb 2C5), which specifically recognizes and binds to live cancer cells, was conjugated to the surface of long-circulating PEGylated liposomes loaded with an 111In complex [47]. The whole-body direct gamma-imaging of tumor bearing mice showed a superior in vivo tumor accumulation of the targeted liposomes compared to non-targeted control formulations. These studies demonstrate that liposomes are useful to develop radionuclide-based nanotheranostics.

\subsection{Liposome-based therapeutics and fluo- rescence imaging}

Fluorescence imaging techniques using fluorescent molecules or QDs are important tools in studying molecular biology and nanomedicine [48-50]. A number of these techniques have been applied in developing liposomes for applications in nanotheranostics. Although a plethora of fluorescent compounds are available, those emitting near infrared (NIR) fluorescence are particularly desirable owing to better tissue penetration and less interference from tissue auto-fluorescence compared to emissions at a lower wavelength. Lowery et al. have conjugated AlexaFluor-750 to the surface of cholesterol/DSPC liposomes for NIR imaging [51]. In this study, the labeling of liposomes with florescent molecules assisted the evaluation of a peptide, HVGGSSV, in targeting tumors for the delivery of DOX. NIR imaging performed on C57/BL6 nude mice implanted with Lewis lung carcinoma and human lung cancer H460 cells showed that targeted liposomes preferentially accumulated within the irradiated tumors when compared with the non-targeted liposomes. Labeling with AlexaFluor-750 also helped to evaluate the pharmacokinetics of the nanotheranostic liposomes, which showed a longer circulation half-life and a higher accumulation and retention within tumors than those of the free drugs, leading to improved anti-cancer efficacy.

Developing liposome-based nanotheranostics with QD-based fluorescence imaging requires the hybridization of QD with liposomes, which can be accomplished by modifying the physicochemical properties, particularly surface properties, of the QDs. For example, QDs capped by trioctylphosphine oxide showed a hydrophobic nature and the incorporation of QD took place within the lipid bilayers [52]. The internal aqueous phase of liposomes can encapsulate hydrophilic drugs and their release in part depends on the type of lipids used. In another study, Al-Jamal 
et al. have developed a strategy to encapsulate PEG-lipid-coated QD ( $f$-QD) into the aqueous cores of liposome vesicles [53]. By varying the type and composition of lipids, formulation parameters such as liposome surface charge, QD surface coating and lipid rigidity were explored to formulate stable hybrid liposomes. Furthermore, NIR imaging provided by the encapsulated $f$-QD allowed for in vivo characterization of pharmacokinetics of the liposomes in C57BL6 mice bearing B16F10 melanoma tumor, which was further confirmed by additional techniques including inductively coupled plasma mass spectrometry and ${ }^{14} \mathrm{C}$ radio tracing. These studies suggest that drug-loaded, QD-based liposomes constitute promising multifunctional delivery vehicles capable of theranostic applications.

\section{Polymer-based nanotheranostics}

Polymeric NPs are a class of nanocarriers established for numerous drug delivery applications [54]. Polymeric NPs can be formulated by conjugating multiple functional units to soluble macromolecules; alternatively, they can be made by co-polymer self-assembly. In a typical self-assembled formulation, polymeric NP cores can be loaded with a variety of therapeutic or imaging agents, where surface or bulk erosion, diffusion through the polymer matrix, swelling followed by diffusion, or stimulation by the local environment results in the sustained and controlled release of these agents [24]. In addition, conjugation of drug molecules to the polymer backbone allows for precise drug loading with additional control over drug release profiles [55]. To ensure stability while minimizing immunogenicity, polymeric cores are shielded by stealth materials such PEG. Targeting moieties can be further introduced to the surface. Polymers derived from natural resources such as chitosan and cyclodextrin have received extensive attention in developing biocompatible polymeric NPs [56]. Meanwhile, a large collection of synthetic polymers with outstanding biocompatibility and biodegradability has also been available to formulate polymeric NPs [57-60]. Increasingly, polymer-based NPs have also attracted much attention in developing theranostic platforms.

\section{I. Polymer-based therapeutics and MRI}

Similar to liposomes, polymeric NPs have been shown to be effective carriers for MRI contrast agents such as SPIOs and Gd-based compounds [61-63]. For example, the mixture of SPIOs and DOX can be directly encapsulated by using amphiphilic block co-polymer composed of maleimide-PEG-poly(lactic acid) (PLA), which self-assembles to form polymeric
NP-based nanotheranostics for both drug delivery and MRI. In two independent studies, surface-presenting maleimide groups allow for the conjugation of either cRGD molecules to target $a_{v} \beta_{3}$ integrins [64] or short peptides containing 10 amino acids to target $a_{v} \beta_{6}$ integrins [65]. The resultant nanotheranostic particles allowed for the evaluation of pharmacokinetics by MRI in real-time. In tumor-bearing mice, targeted NPs showed a higher tumor accumulation when compared with non-targeted NPs owing to the integrin-mediated endocytosis, which further led to enhanced tumor retardation. Studies using other polymers such as Pluronic ${ }^{\circledR}$ F-127 have also resulted in stable formulations capable of simultaneous imaging and therapy, suggesting that the strategy of encapsulating SPIO-DOX mixture to formulate nanotheranostic particles is applicable to many existing polymeric NP systems [66].

Gadolinium compounds have also been used to formulate polymeric NP-based theranostic formulations for real-time visualization of blood circulation, biodistribution, and tumor accumulation. Ye et al. have conjugated gadolinium- (1,4,7,10-tetraazacyclododecane-1,4,7-trisacetic acid) (Gd-DO3A) to the side chains of poly(L-glutamic acid) (PGA) and subsequently fractionated the polymer-GD-DO3A conjugates into three groups with molecular weights of 87,50 and $28 \mathrm{kDa}$ (Figure 3) [67]. In mice bearing MDA-MB-231 human breast cancer xenografts, strong MR signal enhancement was observed in the tumor periphery. Further pharmacokinetic studies revealed that the conjugates with higher molecular weights $(87$ and $50 \mathrm{kDa}$ ) exhibited a more prolonged blood circulation and higher tumor accumulation, although the difference between the conjugates of 87 and $50 \mathrm{kDa}$ was not significant. In contrast, the conjugate of 28 $\mathrm{kDa}$ was rapidly cleared from the circulation and had a relatively lower tumor accumulation. This observation again confirms that it is beneficial to use carriers within a certain nanometer size range for tumor targeting as opposed to the use of small molecules. In another study, Gd complexed together with DOX and folic acid as a targeting ligand were conjugated to a cyclodextrin-based star copolymer, resulting in long-circulating NP formulations with considerable MR contrast enhancement [68]. The advantage of this system is the asymmetric structure of the star copolymer, which accommodates multiple reactive groups for sequential conjugations of different moieties leading to better spatiotemporal-controlled delivery of cargo. In particular, the conjugation of DOX to the star copolymer through a $\mathrm{pH}$-sensitive linker further improved the efficacy. 


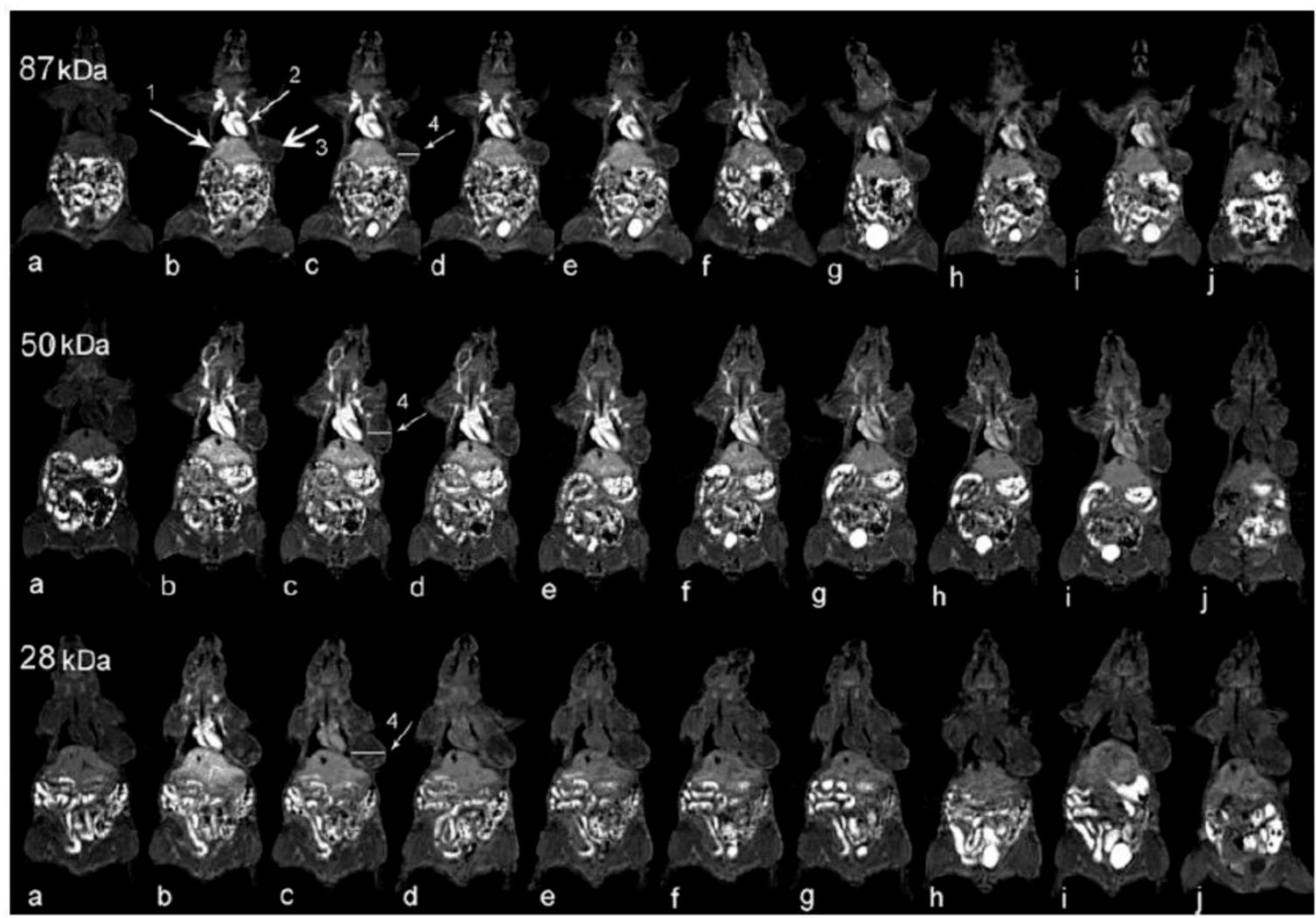

Figure 3. Coronal MR images of tumor bearing mice (a) before and at (b) I, (c) II, (d) 20, (e) 30, (f) 60, (g) I20, (h) I80, and (i) 240 minutes and (j) 24 hours after injection of PGA-I,6-hexanediamine-(Gd-DO3A) conjugates of different molecular weights. Arrows point to the (I) liver, (2) heart, and (3) tumor tissue, and (4) the cross section is for subsequent quantitative analysis. [67]

\subsection{Polymer-based therapeutics and radionu- clide imaging}

Polymer-based NP formulations have been explored as carriers for radionuclide imaging. For example, by conjugating radionuclide compounds including ${ }^{11} \mathrm{C},{ }^{18} \mathrm{~F},{ }^{64} \mathrm{Cu},{ }^{76} \mathrm{Br},{ }^{99} \mathrm{mTc},{ }^{111} \mathrm{In}$ and ${ }^{90} \mathrm{Y}$, researchers have extensively explored a wide range of copolymers such as $N$-(2-hydroxypropyl) methacrylamide (HPMA) to formulate robust nano-sized delivery systems [69]. Nanotheranostic platforms combining therapeutic molecules and radionuclide compounds have also been developed. For example, Mitra, et al. have conjugated ${ }^{111}$ In compounds to HPMA for scintigraphic imaging in an attempt to optimize HPMA-based formulations when functionalized by mono-(RGDfk) and doubly cyclized (RGD4C), two $a_{v} \beta_{3}$-binding peptides with high stability. In vitro studies using human umbilical vein endothelial cells (HUVECs) demonstrated that the polymer conjugates were able to target the integrins, resulting in enhanced cell uptake. In C57BL/6NHsd mice bearing Lewis lung carcinoma, both RGDfk and RGD4C enhanced the tumor localization of the conjugates, with further contrast enhancement observed at the tumor site after 24 hours of injection.

To develop effective nanotheranostic platforms, the potential of adding PET imaging agents into polymer-based nanocarriers, particularly used as non-invasive visualization and quantification of angiogenic biomarkers, has been realized [70]. For example, Chen, et al have coupled c(RGDyK), a cyclic RGD peptide, to ${ }^{64} \mathrm{Cu}$-DOTA through a PEG linker and the resulting conjugates showed effective targeting in brain tumor models [71]. In addition, PEG molecules used to functionalize SPIOs also provide additional positions to conjugate both tumor-targeting ligands such as c(RGDfC) peptide and PET imaging agents such as ${ }^{64} \mathrm{Cu}$ chelators, a useful strategy to combine drug delivery to tumors with PET/MRI dual-modality imaging [72]. Notably, developments built on these pilot studies can be extended for targeted internal radiotherapy via loading the same platform with therapeutic dosages of radionuclides.

\subsection{Polymer-based therapeutics and fluores- cence imaging}

By integrating fluorescence imaging techniques into polymeric NPs, image-guided drug delivery al- 
lows researchers to monitor drug pharmacokinetics, intratumoral drug distribution, and drug tumor accumulation in real-time [19]. For example, Peng et al. have developed multifunctional polymeric NPs composed of PEG-polycaprolactone (PCL) di-block co-polymer to load IR-780, a NIR florescent dye, for both NIR imaging and photodynamic therapy (PDT) (Figure 4) [73]. The NPs were also labeled with ${ }^{188} \mathrm{Re}$ for microSPECT-guided tumor imaging. In BALB/c athymic nude mice bearing HCT-116 colorectal carcinoma, a preferential tumor accumulation was observed and the addition of NIR irradiation enhanced tumor inhibition when compared with control groups treated with PBS, NIR irradiation only, or micelles only.

In another study, Lee et al. have used glycol chitosan NPs to load chlorin e6 (Ce6), a photosensitizer that allows for both in vivo imaging and PDT (Figure 5) [74]. Compared with physical encapsulation, chemical conjugation of Ce6 to amphiphilic glycol chitosan- $5 \beta$-cholanic acid resulted in a formulation with a more sustained release profile of $\mathrm{Ce} 6$, a longer circulation half-life, and a higher tumor accumulation. In vivo NIR imaging of mice bearing HT-29 human colon adenocarcinoma showed clearly the tumor lo- calization with a low interference from surrounding normal tissues. The combination of NP formulation and the subsequent laser irradiation exhibited severe necrosis of the tumor tissue and decreased tumor volume, while free Ce6 and NPs with physically encapsulated Ce6 failed to show noticeable phototoxicity in the tumor tissues. In PDT, the interaction between the photosensitizers and the tumor cells also affect the efficacy, as short-lived radicals generated from the photosensitizers are only toxic to cells or tissues in close proximity. To understand this effect, (poly(N-vinyl caprolactam)-g-PLA, a pH-sensitive copolymer with endosomolytic ability, and poly(N-vinyl caprolactam-co-N-vinyl imidazole)-g-PLA), a non-pH-sensitive copolymer, were synthesized and utilized for the encapsulation of protoporphyrin IX (PPIX) for in vitro and in vivo PDT studies [75]. With $\mathrm{pH}$-sensitive particles, PPIX was found in the nucleus; in contrast, PPIX was largely trapped in the lysosomes for the non- $\mathrm{pH}$ sensitive particles. In female BALB/c nude mice bearing A549 cells xenografts, NP-based PDT showed a better regression of tumor growth than free PPIX; and effective PDT-induced inhibition of tumor growth was only observed in mice treated with $\mathrm{pH}$-sensitive NPs.
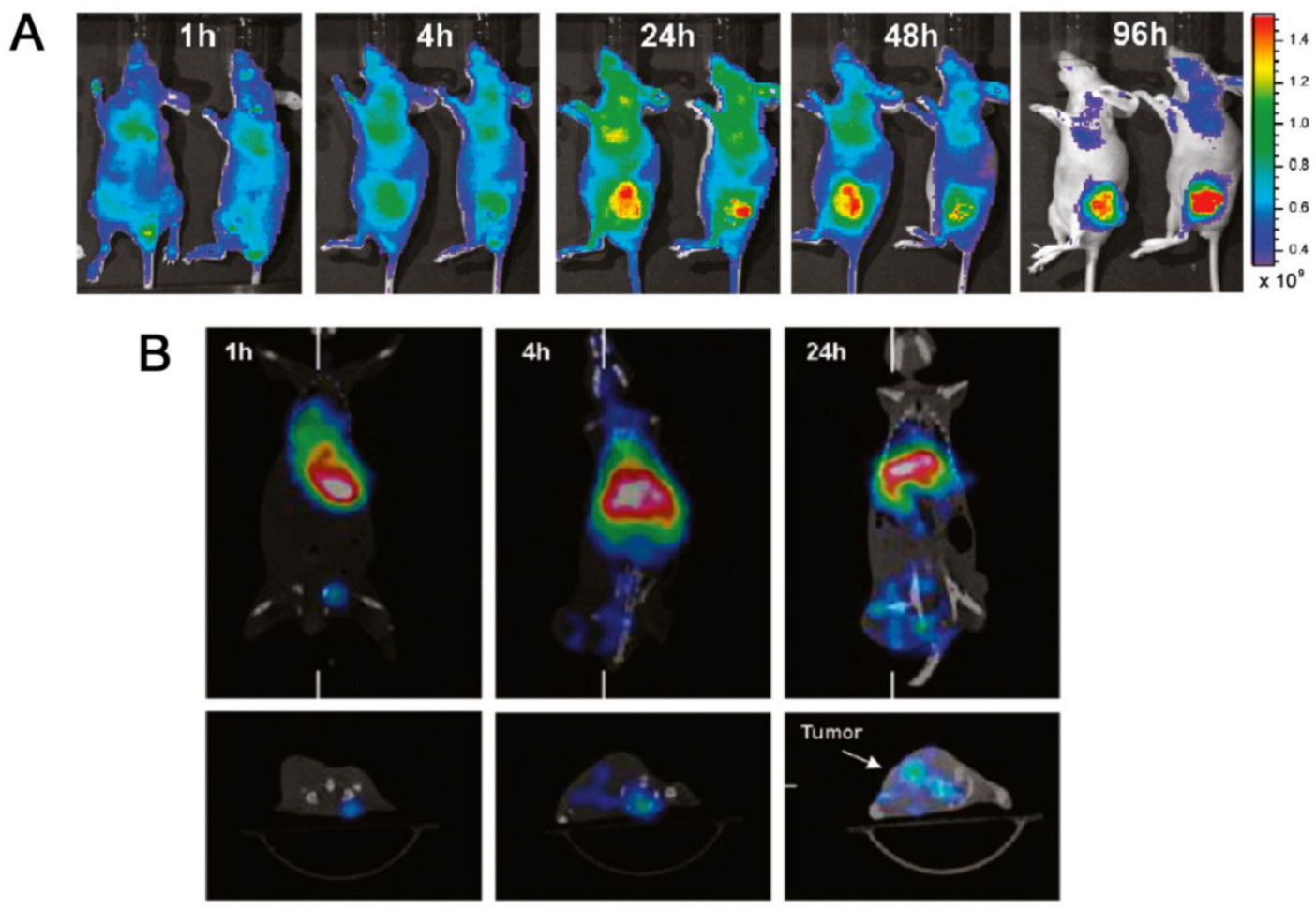

Figure 4. Multifunctional polymeric nanoparticles composed of PEG-PCL di-block co-polymer were loaded with IR-780, a NIR florescent dye, for both NIR imaging and photodynamic therapy (PDT). The NPs were also labeled with 188 Re for microSPECT-guided tumor imaging. (A) Time-lapse near-IR fluorescence images of mice bearing HCT-II6 tumors after intravenous injections of PEG-PCL polymeric NPs containing IR-780. (B) MicroSPECT/CT images were obtained by first injecting I88Re-labeled IR-780 particles and then imaging at I, 4, and 24 hours later, respectively. [73] 


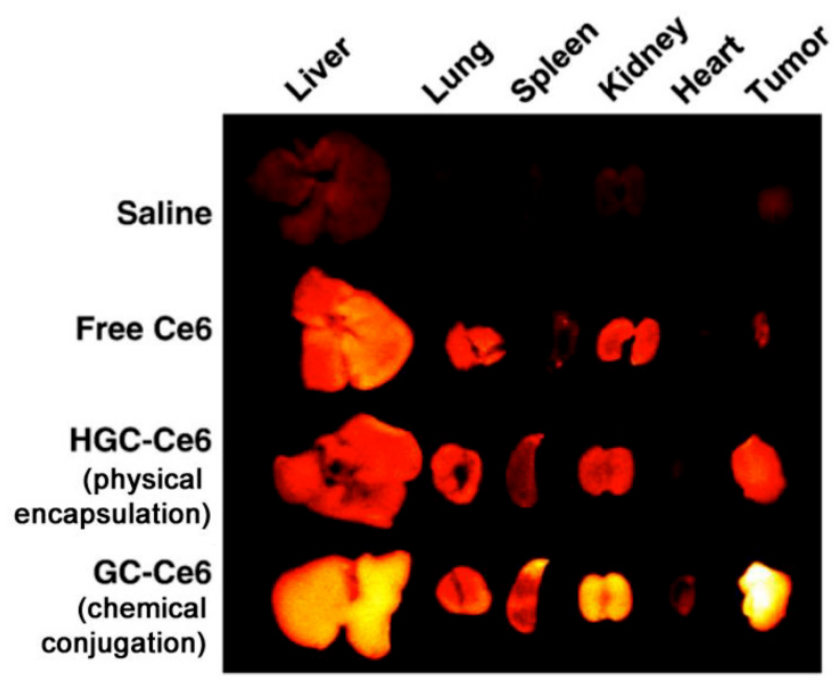

Figure 5. Athymic nude mice bearing HT-29 tumors after intravenous injection of saline, free Ce6, HGC-Ce6 (a formulation where Ce6 was physically encapsulated), or GC-Ce6 (a formulation where Ce6 was chemically conjugated). Ce6 dosage is $2.5 \mathrm{mg} / \mathrm{kg}$. Here the figure shows ex vivo images of normal organs (liver, lung, spleen, kidney, and heart) and tumors excised at 2 days post-injection of saline, free Ce6, HGC-Ce6, or GC-Ce6. [74]

\section{Conclusions}

Liposomes and polymer-based NPs are both established drug delivery platforms for cancer treatment. The desire to combine both therapeutics and imaging into a single carrier has advanced these delivery systems one step further, resulting in a number of novel theranostic platforms. Extensive knowledge learned from the development of multifunctional therapeutic nanocarriers will continue to inspire researchers to engineer lipid- and polymer-based nanotherapeutic platforms with multiple imaging modalities incorporated into increasingly sophisticated architectures [76]. Meanwhile, the rapid development of nanomedicine has extended NP therapeutics beyond oncological applications into other fields, particularly cardiology [77, 78] and tissue engineering [79], where we believe that nanotherapeutic platforms will find numerous potential applications. While these technological advances continually progress toward the clinical translation, we envision that basic research aimed to gain deeper understandings on imaging agents, particularly on the connections between their imaging capability and their physicochemical microenvironments within nanocarriers, will play a key role in developing robust nanotherapeutic platforms with high-performance imaging capability. Taken collectively, these new developments will bring great clinical potential and a bright future for nanotherapeutics.

\section{Acknowledgements}

This work is supported by the National Science

\section{Foundation Grants DMR-1216461 and} CMMI-1031239.

\section{Competing Interests}

The authors have declared that no competing interest exists.

\section{References}

[1] Morgan B. Opportunities and pitfalls of cancer imaging in clinical trials. Nat Rev Clin Oncol. 2011; 8: 517-27.

[2] Sumer B, Gao JM. Theranostic nanomedicine for cancer. Nanomedicine. 2008; 3: 137-40.

[3] Kievit FM, Zhang M. Cancer nanotheranostics: Improving imaging and therapy by targeted delivery across biological barriers. Adv Mater. 2011; 23: H217-H47.

[4] Sun D. Nanotheranostics: Integration of imaging and targeted drug delivery. Mol Pharm. 2010; 7: 1879.

[5] Davis ME, Chen Z, Shin DM. Nanoparticle therapeutics: an emerging treatment modality for cancer. Nat Rev Drug Discov. 2008; 7: 771-82.

[6] Lammers T, Kiessling F, Hennink WE, Storm G. Nanotheranostics and image-guided drug delivery: Current concepts and future directions. Mol Pharm. 2010; 7: 1899-912.

[7] Willmann JK, van Bruggen N, Dinkelborg LM, Gambhir SS. Molecular imaging in drug development. Nat Rev Drug Discov. 2008; 7: 591-607.

[8] Davis ME, Chen Z, Shin DM. Nanoparticle therapeutics: an emerging treatment modality for cancer. Nat Rev Drug Discov. 2008; 7: 771-82.

[9] Petros RA, DeSimone JM. Strategies in the design of nanoparticles for therapeutic applications. Nat Rev Drug Discov. 2010; 9: 615-27.

[10] Wang AZ, Langer R, Farokhzad OC. Nanoparticle delivery of cancer drugs. Ann Rev Med. 2012; 63: 185-98.

[11] Farokhzad OC, Langer R. Impact of nanotechnology on drug delivery. ACS Nano. 2009; 3: 16-20.

[12] Timko BP, Whitehead K, Gao W, Kohane DS, Farokhzad O, Anderson D, et al. Advances in drug delivery. Ann. Rev. Mater. Res. 2011; 41: 1-20.

[13] Safra T, Muggia F, Jeffers S, Tsao-Wei DD, Groshen S, Lyass O, et al. Pegylated liposomal doxorubicin (doxil): Reduced clinical cardiotoxicity in patients reaching or exceeding cumulative doses of $500 \mathrm{mg} / \mathrm{m}(2)$. Ann Oncol. 2000; 11: 1029-33.

[14] Hofheinz RD, Gnad-Vogt SU, Beyer U, Hochhaus A. Liposomal encapsulated anti-cancer drugs. Anti-Cancer Drug. 2005; 16: 691-707.

[15] Ning YM, He K, Dagher R, Sridhara R, Farrell AT, Justice R, et al. Liposomal doxorubicin in combination with bortezomib for relapsed or refractory multiple myeloma. Oncology (Williston Park). 2007; 21: 1503-8. 
[16] Zhang L, Gu FX, Chan JM, Wang AZ, Langer RS, Farokhzad OC. Nanoparticles in medicine: Therapeutic applications and developments. Clin Pharm Ther. 2008; 83: 761-9.

[17] Na HB, Song IC, Hyeon T. Inorganic nanoparticles for MRI contrast agents. Adv Mater. 2009; 21: 2133-48.

[18] Gambhir SS. Molecular imaging of cancer with positron emission tomography. Nat Rev Cancer. 2002; 2: 683-93.

[19] Kobayashi H, Ogawa M, Alford R, Choyke PL, Urano Y. New strategies for fluorescent probe design in medical diagnostic imaging. Chem Rev. 2010; 110: 2620-40.

[20] Rahmim A, Zaidi H. PET versus SPECT: strengths, limitations and challenges. Nucl Med Comm. 2008; 29: 193-207.

[21] Kelkar SS, Reineke TM. Theranostics: Combining imaging and therapy. Bioconj Chem. 2011; 22: 1879-903.

[22] Malam Y, Loizidou M, Seifalian AM. Liposomes and nanoparticles: nanosized vehicles for drug delivery in cancer. Trends Pharmacol Sci. 2009; 30: 592-9.

[23] Ferrari M. Cancer nanotechnology: opportunities and challenges. Nat Rev Cancer. 2005; 5: 161-71.

[24] Peer D, Karp JM, Hong S, FaroKHzad OC, Margalit R, Langer R. Nanocarriers as an emerging platform for cancer therapy. Nat Nanotechnol. 2007; 2: 751-60.

[25] Sharma A, Sharma US. Liposomes in drug delivery: progress and limitations. Int J Pharm. 1997; 154: 123-40.

[26] Barenholz Y. Liposome application: problems and prospects. Curr Opin Colloid Interface. 2001; 6: 66-77.

[27] Martina MS, Fortin JP, Menager C, Clement O, Barratt G, Grabielle-Madelmont $\mathrm{C}$, et al. Generation of superparamagnetic liposomes revealed as highly efficient MRI contrast agents for in vivo imaging. J Am Chem Soc. 2005; 127: 10676-85.

[28] Shapiro EM, Skrtic S, Sharer K, Hill JM, Dunbar CE, Koretsky AP. MRI detection of single particles for cellular imaging. Proc Natl Acad Sci USA. 2004; 101: 10901-6.

[29] Wang YXJ, Hussain SM, Krestin GP. Superparamagnetic iron oxide contrast agents: physicochemical characteristics and applications in MR imaging. Eur Radiol. 2001; 11: 2319-31.

[30] Kabalka GW, Davis MA, Moss TH, Buonocore E, Hubner K, Holmberg E, et al. Gadolinium-labeled liposomes containing various amphiphilic Gd-Dtpa derivatives - targeted MRI contrast enhancement agents for the liver. Magnet Reson Med. 1991; 19: 406-15.

[31] Viglianti BL, Abraham SA, Michelich CR, Yarmolenko PS, MacFall JR, Bally $\mathrm{MB}$, et al. In vivo monitoring of tissue pharmacokinetics of liposome/drug using MRI: Illustration of targeted delivery. Magnet Reson Med. 2004; 51: 1153-62.

[32] Erten A, Wrasidlo W, Scadeng M, Esener S, Hoffman RM, Bouvet M, et al. Magnetic resonance and fluorescence imaging of doxorubicin-loaded nanoparticles using a novel in vivo model. Nanomedicine. 2010; 6: 797-807.

[33] Tai LA, Tsai PJ, Wang YC, Wang YJ, Lo LW, Yang CS. Thermosensitive liposomes entrapping iron oxide nanoparticles for controllable drug release. Nanotechnology. 2009; 20: 135101.

[34] Tagami T, Foltz WD, Ernsting MJ, Lee CM, Tannock IF, May JP, et al. MRI monitoring of intratumoral drug delivery and prediction of the therapeutic effect with a multifunctional thermosensitive liposome. Biomaterials. 2011; 32: 6570-8

[35] Kono K, Nakashima S, Kokuryo D, Aoki I, Shimomoto H, Aoshima S, et al. Multi-functional liposomes having temperature-triggered release and magnetic resonance imaging for tumor-specific chemotherapy. Biomaterials. 2011; 32: 1387-95.

[36] Soundararajan A, Bao A, Phillips WT, Perez R, Goins BA. [(186)Re]Liposomal doxorubicin (Doxil): in vitro stability, pharmacokinetics, imaging and biodistribution in a head and neck squamous cell carcinoma xenograft model. Nucl Med Biol. 2009; 36: 515-24.

[37] Bao A, Goins B, Klipper R, Negrete G, Phillips WT. Re-186-liposome labeling using Re-186-SNS/S complexes: In vitro stability, imaging, and biodistribution in rats. J Nucl Med. 2003; 44: 1992-9.

[38] Bao A, Phillips W, Goins B, Otto R. Tumor brachytherapy using intratumoral injection of beta-emitting therapeutic radionuclides carried within nanoparticles. Med Phys. 2006; 33: 2236-7.

[39] Chen LC, Chang CH, Yu CY, Chang YJ, Wu YH, Lee WC, et al. Pharmacokinetics, micro-SPECT/CT imaging and therapeutic efficacy of (188)Re-DXR-liposome in C26 colon carcinoma ascites mice model. Nucl Med Biol. 2008; 35: 883-93.

[40] Osborne MP, Richardson VJ, Jeyasingh K, Ryman BE. Radionuclide-labeled liposomes - a new lymph node imaging agent. Int J Nucl Med Biol. 1979; 6: 75-83.
[41] Harrington KJ, Mohammadtaghi S, Uster PS, Glass D, Peters AM, Vile RG, et al. Effective targeting of solid tumors in patients with locally advanced cancers by radiolabeled PEGylated liposomes. Clin Cancer Res. 2001; $7: 243-54$

[42] Hoefnagel CA. Radionuclide cancer therapy. Ann Nucl Med. 1998; 12: $61-70$.

[43] Zweit J. Radionuclides and carrier molecules for therapy. Phys Med Biol. 1996; 41: 1905-14

[44] Kostarelos K, Emfietzoglou D. Tissue dosimetry of liposome-radionuclide complexes for internal radiotherapy: Toward liposome-targeted therapeutic radiopharmaceuticals. Anticancer Res. 2000; 20: $3339-45$.

[45] Kostarelos K, Emfietzoglou D, Stamatelou M. Liposome-mediated delivery of radionuclides to tumor models for cancer radiotherapy: A quantitative analysis. J Liposome Res. 1999; 9: 407-24.

[46] Petersen AL, Binderup T, Rasmussen P, Henriksen JR, Elema DR, Kjaer A, et al. (64)Cu loaded liposomes as positron emission tomography imaging agents. Biomaterials. 2011; 32: 2334-41.

[47] Erdogan S, Roby A, Torchilin VP. Enhanced tumor visualization by gamma-scintigraphy with (111)In-labeled polychelating-polymer-containing immunoliposomes. Mol Pharma. 2006; 3: 525-30.

[48] Mulder WJM, Strijkers GJ, Habets JW, Bleeker EJW, van der Schaft DWJ, Storm G, et al. MR molecular imaging and fluorescence microscopy for identification of activated tumor endothelium using a bimodal lipidic nanoparticle. Faseb J. 2005; 19: 2008-10.

[49] Saito R, Krauze MT, Bringas JR, Noble C, McKnight TR, Jackson P, et al. Gadolinium-loaded liposomes allow for real-time magnetic resonance imaging of convection-enhanced delivery in the primate brain. Exp Neurol. 2005; 196: 381-9.

[50] Weissleder R, Tung CH, Mahmood U, Bogdanov A. In vivo imaging of tumors with protease-activated near-infrared fluorescent probes. Nat Biotechnol. 1999; 17: 375-8.

[51] Lowery A, Onishko H, Hallahan DE, Han ZZ. Tumor-targeted delivery of liposome-encapsulated doxorubicin by use of a peptide that selectively binds to irradiated tumors. J Control Release. 2011; 150: 117-24.

[52] Tian B, Al-Jamal WT, Al-Jamal KT, Kostarelos K. Doxorubicin-loaded lipid-quantum dot hybrids: Surface topography and release properties. Int J Pharm. 2011; 416: 443-7.

[53] All-Jamal WT, Al-Jamal KT, Tian B, Cakebread A, Halket JM, Kostarelos $\mathrm{K}$. Tumor targeting of functionalized quantum dot-liposome hybrids by intravenous administration. Mol Pharma. 2009; 6: 520-30.

[54] Fang RH, Zhang L. Dispersion-based methods for the engineering and manufacture of polymeric nanoparticles for drug delivery applications. J Nanoeng Nanomanuf. 2011; 1: 106-12.

[55] Aryal S, Hu CMJ, Zhang LF. Polymeric nanoparticles with precise ratiometric control over drug loading for combination therapy. Mol Pharm. 2011; 8: 1401-7.

[56] Liu ZH, Jiao YP, Wang YF, Zhou CR, Zhang ZY. Polysaccharides-based nanoparticles as drug delivery systems. Adv Drug Deliver Rev. 2008; 60: 1650-62.

[57] $\mathrm{Hu} \mathrm{KL}, \mathrm{Li}$ JW, Shen YH, Lu W, Gao XL, Zhang QZ, et al. Lactoferrin-conjugated PEG-PLA nanoparticles with improved brain delivery: In vitro and in vivo evaluations. J Control Release. 2009; 134: 55-61.

[58] Cheng J, Teply BA, Sherifi I, Sung J, Luther G, Gu FX, et al. Formulation of functionalized PLGA-PEG nanoparticles for in vivo targeted drug delivery. Biomaterials. 2007; 28: 869-76.

[59] Kabanov AV, Chekhonin VP, Alakhov VY, Batrakova EV, Lebedev AS, Meliknubarov NS, et al. The neuroleptic activity of haloperidol increases after its solubilization in surfactant micelles - micelles as microcontainers for drug targeting. Febs Lett. 1989; 258: 343-5.

[60] Miller DW, Batrakova EV, Waltner TO, Alakhov VY, Kabanov AV. Interactions of pluronic block copolymers with brain microvessel endothelial cells: Evidence of two potential pathways for drug absorption. Bioconjugate Chem. 1997; 8: 649-57.

[61] Nitin N, LaConte LEW, Zurkiya O, Hu X, Bao G. Functionalization and peptide-based delivery of magnetic nanoparticles as an intracellular MRI contrast agent. J Biol Inorg Chem. 2004; 9: 706-12.

[62] Andre JP, Toth E, Fischer H, Seelig A, Macke HR, Merbach AE. High relaxivity for monomeric Gd(DOTA)-based MRI contrast agents, thanks to micellar self-organization. Chem-Eur J. 1999; 5: 2977-83.

[63] Zhang GD, Zhang R, Wen XX, Li L, Li C. Micelles based on biodegradable poly(L-glutamic acid)-b-polylactide with paramagnetic gd ions chelated to the shell layer as a potential nanoscale IVIRI-visible delivery system. Biomacromol. 2008; 9: 36-42.

[64] Nasongkla N, Bey E, Ren JM, Ai H, Khemtong C, Guthi JS, et al. Multifunctional polymeric micelles as cancer-targeted, MRI-ultrasensitive drug delivery systems. Nano Lett. 2006; 6: 2427-30. 
[65] Guthi JS, Yang SG, Huang G, Li SZ, Khemtong C, Kessinger CW, et al. MRI-visible micellar nanomedicine for targeted drug delivery to lung cancer cells. Mol Pharm. 2010; 7: 32-40.

[66] Lai JR, Chang YW, Yen HC, Yuan NY, Liao MY, Hsu CY, et al. Multifunctional doxorubicin/superparamagnetic iron oxide-encapsulated Pluronic F127 micelles used for chemotherapy/magnetic resonance imaging. J Appl Phys. 2010; 107: 09B318.

[67] Ye FR, Ke TY, Jeong EK, Wang XL, Sung YG, Johnson M, et al. Noninvasive visualization of in vivo drug delivery of poly(L-glutamic acid) using contrast-enhanced MRI. Mol Pharmaceut. 2006; 3: 507-15.

[68] Liu T, Li X, Qian Y, Hu X, Liu S. Multifunctional pH-Disintegrable micellar nanoparticles of asymmetrically functionalized beta-cyclodextrin-Based star copolymer covalently conjugated with doxorubicin and DOTA-Gd moieties. Biomaterials. 2012; 33: 2521-31.

[69] Lu Z-R. Molecular imaging of HPMA copolymers: Visualizing drug delivery in cell, mouse and man. Adv Drug Deliver Rev. 2010; 62: 246-57.

[70] Welch MJ, Hawker CJ, Wooley KL. The Advantages of Nanoparticles for PET. J Nucl Med. 2009; 50: 1743-6.

[71] Chen XY, Hou YP, Tohme M, Park R, Khankaldyyan V, Gonzales-Gomez I, et al. Pegylated Arg-Gly-Asp peptide: Cu-64 labeling and PET imaging of brain tumor alpha(v)beta(3)-integrin expression. J Nucl Med. 2004; 45: 1776-83.

[72] Yang X, Hong H, Grailer JJ, Rowland IJ, Javadi A, Hurley SA, et al. cRGD-functionalized, DOX-conjugated, and (64)Cu-labeled superparamagnetic iron oxide nanoparticles for targeted anticancer drug delivery and PET/MR imaging. Biomaterials. 2011; 32: 4151-60.

[73] Peng CL, Shih YH, Lee PC, Hsieh TMH, Luo TY, Shieh MJ. Multimodal image-guided photothermal therapy mediated by (188)Re-labeled micelles containing a cyanine-type photosensitizer. ACS Nano. 2011; 5: 5594-607.

[74] Lee SJ, Koo H, Jeong H, Huh MS, Choi Y, Jeong SY, et al. Comparative study of photosensitizer loaded and conjugated glycol chitosan nanoparticles for cancer therapy. J Control Release. 2011; 152: 21-9.

[75] Tsai H-C, Tsai C-H, Lin S-Y, Jhang C-R, Chiang Y-S, Hsiue G-H. Stimulated release of photosensitizers from graft and diblock micelles for photodynamic therapy. Biomaterials. 2012; 33: 1827-37.

[76] Park JH, von Maltzahn G, Ruoslahti E, Bhatia SN, Sailor MJ. Micellar hybrid nanoparticles for simultaneous magnetofluorescent imaging and drug delivery. Angew Chem Int Ed. 2008; 47: 7284-8.

[77] Lobatto ME, Fuster V, Fayad ZA, Mulder WJM. Perspectives and opportunities for nanomedicine in the management of atherosclerosis. Nat Rev Drug Discov. 2011; 10: 835-52.

[78] Chan JM, Rhee J-W, Drum CL, Bronson RT, Golomb G, Langer R, et al. In vivo prevention of arterial restenosis with paclitaxel-encapsulated targeted lipid-polymeric nanoparticles. Proc Natl Acad Sci USA. 2011; 108 : 19347-52.

[79] Dvir T, Timko BP, Kohane DS, Langer R. Nanotechnological strategies for engineering complex tissues. Nat Nanotechnol. 2011; 6: 13-22. 